\title{
Predictors of Prolonged Laparoscopic Radical Prostatectomy and the Creation of a Scoring System for the Duration
}

This article was published in the following Dove Press journal: Cancer Management and Research

\author{
Shao-Hao Chen ${ }^{1} *$ \\ Zhi-Bin Ke ${ }^{\mathrm{I} *}$ \\ Yu-Peng $\mathrm{Wu}^{\mathrm{l}, *}$ \\ Dong-Ning Chen ${ }^{1} *$ \\ Xiang Yu' \\ Yu Chen ${ }^{2,3}$ \\ Yong Wei (1D) \\ Qing-Shui Zheng' \\ Xue-Yi Xue (iD ${ }^{\prime}$ \\ Ning $X u(D)$ \\ 'Department of Urology, The First \\ Affiliated Hospital of Fujian Medical \\ University, Fuzhou 350005, People's \\ Republic of China; ${ }^{2}$ Cancer Bio- \\ Immunotherapy Center, Fujian Medical \\ University Cancer Hospital and Fujian \\ Cancer Hospital, Fuzhou, People's \\ Republic of China; ${ }^{3}$ Department of \\ Medical Oncology, Fujian Medical \\ University Cancer Hospital and Fujian \\ Cancer Hospital, Fuzhou, People's \\ Republic of China
}

*These authors contributed equally to this work
Correspondence: Ning Xu; Xue-Yi Xue Department of Urology, The First Affiliated Hospital of Fujian Medical University, 20 Chazhong Road, Fuzhou 350005 , People's Republic of China Tel +86-5918798I687

Email drxun@fjmu.edu.cn;

xuexueyi@fjmu.edu.cn
Purpose: To identify the relevant factors, and create and validate a predictive scoring system for the duration of laparoscopic radical prostatectomy (LRP).

Patients and Methods: We retrospectively analyzed clinicopathological data from 436 patients who underwent LRP between January 2014 and January 2019, of whom 304 cases were used as a model creation group and 132 were used as a validation group. Uni/multivariate linear regression analysis was performed to determine the predictors of the duration of the procedure and a novel scoring system was created using these predictors. External validation of the scoring system was performed. The Hosmer-Lemeshow test was used to determine the goodness-of-fit of the model and calibration plots were created for visual assessment.

Results: "Prolonged duration" was defined as a duration of the procedure that was longer than the mean (>150 min) duration. Multivariate analysis showed that body mass index (BMI), prostate volume, intravesicular protrusion of the prostate (IPP), the ratio of the crosssectional areas of the prostate and the Retzius space (P/R), pelvic lymph node dissection, and neurovascular bundle (NVB) preservation were significant predictors of prolonged duration. A scoring system that included these six parameters was created and the area under the curve achieved during receiver operating characteristic analysis using this scoring system was 0.874 (95\% confidence interval [CI]: 0.836-0.913). The Hosmer-Lemeshow test showed that the scoring system was well calibrated $\left(X^{2}=5.339, P=0.376\right)$. The external validation showed that the model had high predictive accuracy (AUC $=0.835,95 \%$ CI: $0.764-0.906$ ) and goodness-of-fit $\left(\mathrm{X}^{2}=4.401, P=0.493\right)$.

Conclusion: The following factors were significantly associated with prolonged duration of laparoscopic radical prostatectomy: BMI, prostate volume, IPP, P/R, pelvic lymph node dissection, and NVB preservation. The novel scoring system created can be used to accurately predict the duration of the procedure, assess the difficulty of surgery, and improve perioperative efficiency.

Keywords: prostate volume, intravesical protrusion of prostate, predictive factor, pelvic lymph node dissection, neurovascular bundle preservation, prostate cancer

\section{Introduction}

Radical prostatectomy (RP) is considered to be the gold standard treatment for localized prostate cancer. ${ }^{1,2}$ Laparoscopic radical prostatectomy (LRP) is the most popular minimally invasive surgical technique for the treatment of localized prostate cancer in China. ${ }^{3}$ Against a background of limited medical resources and increasing medical demand, improvements in operating room efficiency can shorten 
hospitalization time and increase the turnover of patients, which has both social and economic benefits. ${ }^{4}$ Accurate prediction of the duration of surgery can improve the efficient use of surgical resources, reduce waste and costs, and permit more efficient scheduling of surgery. Knowledge of the predictors of the duration of LRP is essential to achieve this cost reduction, and improve patient care and perioperative efficiency. ${ }^{5}$ Although sometimes the surgical procedures may be prolonged, preoperative knowledge of this is helpful to arrange operating room schedules and allocate staff, to limit the higher cost associated. However, if a procedure takes longer than expected, this not only results in extra overtime payments and increases the cost of the procedure itself, but also affects the performance of the medical staff. ${ }^{6}$ Several previous studies have shown that the frequent necessity for overtime is one of the main reasons for medical staff to resign. ${ }^{7}$ Therefore, prediction of the duration of procedures is a cost-effective consideration for medical staff, patients, hospitals, and society in general.

Previous research has shown that obesity and prostate size are independent predictors of prolonged surgical time with respect to traditional open RP, LRP, and robotassisted laparoscopic prostatectomy (RALP). ${ }^{8-13}$ In addition, several studies have shown that factors such as a lymph node dissection procedure and previous abdominal surgery are associated with prolonged LRP and RALP. ${ }^{14}$ However, there are many factors that can affect LRP, and these previous studies simply describe predictors of prolonged LRP, without providing a more precise estimation of the duration of the procedure in each instance. In the present study, we have retrospectively analyzed the perioperative data of patients who underwent LRP and quantified the anatomical characteristics of the prostate and surrounding organs to identify predictors of the duration of LRP. Using this information, we created a scoring system for the prediction of the duration of LRP.

\section{Patients and Methods}

\section{Patients}

This study was approved by the Ethics Committee of the First Affiliated Hospital of Fujian Medical University (No. $2,015,084)$. All patients provided written informed consent. We retrospectively analyzed data from 482 patients who underwent LRP at our center between January 2014 and January 2019. Three hundred and thirty-one LRPs were performed by the same senior physician, who had previously performed more than 300 similar procedures (Qing-Shui Zheng). The remaining 151 LRPs were performed by another senior physician who had performed more than 200 similar procedures (Yong Wei). The inclusion criteria were as follows: a diagnosis made on the basis of pathological findings following prostatic biopsy, the absence of distant metastasis, and successful completion of the procedure, without any intraoperative complications. The exclusion criteria were as follows: previous history of prostatic surgery, treatment with endocrine therapy or radiotherapy, conversion to laparotomy, and incomplete clinical data. Patients with a history of transurethral resection of the prostate $(n=6)$, intraoperative bladder stone removal $(\mathrm{n}=2)$, neoadjuvant endocrine therapy $(\mathrm{n}=9)$, intraoperative conversion to laparotomy $(\mathrm{n}=1)$, and incomplete follow-up data $(n=28)$ were excluded from the analysis. In total, 304 procedures that were performed by Qing-Shui Zheng were used as the model creation group and 132 procedures that were performed by Yong Wei were used as the validation group. The characteristics of the patients in each group are summarized in Table 1.

\section{Definitions of the Variables}

All of the participants underwent preoperative magnetic resonance imaging (MRI). The cross-sectional area of the Retzius space was defined as the area surrounded by the pubic bone, levator ani, and rectum (Figure 1A). ${ }^{15}$ We also measured the cross-sectional area of the prostate on an MRI image and calculated the ratio of the cross-sectional areas of the prostate and the Retzius space (P/R: prostate/ Retzius space), which reflects the amount of space surrounding the prostate (Figure 1A). The amount of intravesicular protrusion of the prostate (IPP) was defined as the distance from the deepest part of the prostatic protrusion into the bladder to the level of the bladder neck on the sagittal plane of the MRI image (Figure 1B). We also measured various indicators that may reflect the width or depth of the pelvic cavity: the narrowest distance between the tips of the ischial spines (ISD), the widest distance between the ischial tuberosities (ITD) at the pelvic outlet, the anteroposterior diameter of the pelvis at the mid-plane (MAD), and the anteroposterior diameter of the pelvic outlet (OAD). To evaluate the pelvic depth observed by the surgeons during LRP as objectively as possible, we also measured the depth of the prostatic apex (PAD), which was defined as the vertical distance from the closest edge of the pubic symphysis to the distal edge of the prostatic tip on the sagittal plane of the MRI image. 
Table I Characteristics of the Model Creation and Validation Groups

\begin{tabular}{|c|c|c|}
\hline \multirow[t]{2}{*}{ Variables } & \multicolumn{2}{|c|}{ Mean or Median \pm SD or $n(\%)$} \\
\hline & $\begin{array}{l}\text { Model Building } \\
\text { Group }\end{array}$ & $\begin{array}{l}\text { Validation } \\
\text { Group }\end{array}$ \\
\hline Age (year) & $66.78 \pm 6.53$ & $67.30 \pm 7.06$ \\
\hline Prostate volume $(\mathrm{mL})$ & $44.32 \pm 18.34$ & $45.63 \pm|8.6|$ \\
\hline tPSA (ng/mL) & $16.01 \pm \mid 1.81$ & $14.72 \pm 10.56$ \\
\hline BMI $\left(\mathrm{Kg} / \mathrm{m}^{2}\right)$ & $24.12 \pm 2.90$ & $23.88 \pm 3.01$ \\
\hline$P / R$ & $0.68 \pm 0.10$ & $0.67 \pm 0.10$ \\
\hline IPP (mm) & $8.52 \pm 4.27$ & $8.15 \pm 4.53$ \\
\hline ISD (cm) & $9.63 \pm 0.78$ & $9.60 \pm 0.85$ \\
\hline ITD (cm) & $11.55 \pm 0.91$ & $11.52 \pm 0.95$ \\
\hline MAD $(\mathrm{cm})$ & $10.00 \pm 1.61$ & $9.96 \pm 1.44$ \\
\hline OAD $(\mathrm{cm})$ & $8.14 \pm 1.28$ & $8.07 \pm 1.41$ \\
\hline PAD $(\mathrm{cm})$ & $3.06 \pm 0.48$ & $3.03 \pm 0.59$ \\
\hline ISD/PAD & $3.22 \pm 0.57$ & $3.30 \pm 0.77$ \\
\hline \multicolumn{3}{|l|}{ cT Stage } \\
\hline Tlc & $125(4|| 1)$. & $52(39.39)$ \\
\hline $\mathrm{T} 2 \mathrm{a}$ & $42(\mid 3.82)$ & $18(13.64)$ \\
\hline $\mathrm{T} 2 \mathrm{~b}$ & $37(12.17)$ & $15(11.36)$ \\
\hline $\mathrm{T} 2 \mathrm{c}$ & $78(25.66)$ & $35(26.52)$ \\
\hline$\geq \mathrm{T} 3 \mathrm{a}$ & $22(7.24)$ & $12(9.09)$ \\
\hline \multicolumn{3}{|l|}{ ISUP Groupe } \\
\hline Groupe I & $103(27.30)$ & $32(24.24)$ \\
\hline Groupe 2 & $70(24.01)$ & $28(2 \mid .2 I)$ \\
\hline Groupe 3 & $66(22.70)$ & $29(21.97)$ \\
\hline Groupe 4 & $36(13.16)$ & $20(15.15)$ \\
\hline Groupe 5 & $29(12.83)$ & $23(17.42)$ \\
\hline \multicolumn{3}{|l|}{ Hypertension } \\
\hline Yes & I33 (43.75) & $57(43.18)$ \\
\hline No & |7| (56.25) & $75(56.82)$ \\
\hline \multicolumn{3}{|l|}{ Diabetes } \\
\hline Yes & $72(23.68)$ & $27(20.45)$ \\
\hline No & $232(76.32)$ & $105(79.55)$ \\
\hline \multicolumn{3}{|l|}{$\begin{array}{l}\text { Pelvic lymph node } \\
\text { dissection }\end{array}$} \\
\hline Yes & $210(69.08)$ & $94(7 \mid .2 I)$ \\
\hline No & $94(30.92)$ & $38(28.79)$ \\
\hline \multicolumn{3}{|l|}{ NVB preservation } \\
\hline Yes & 214 (70.39) & $86(65.15)$ \\
\hline No & $90(29.61)$ & $46(34.85)$ \\
\hline \multicolumn{3}{|l|}{ Surgical approach } \\
\hline transperitoneal & $77(25.33)$ & $26(19.70)$ \\
\hline extraperitoneal & $227(74.67)$ & $106(80.30)$ \\
\hline \multicolumn{3}{|l|}{$\begin{array}{l}\text { Previous abdominal } \\
\text { surgery }\end{array}$} \\
\hline Yes & $45(14.80)$ & $17(12.88)$ \\
\hline
\end{tabular}

(Continued)
Table I (Continued).

\begin{tabular}{|c|l|l|}
\hline \multirow{2}{*}{ Variables } & \multicolumn{2}{|l|}{ Mean or Median \pm SD or $\mathbf{n}(\%)$} \\
\cline { 2 - 3 } & $\begin{array}{l}\text { Model Building } \\
\text { Group }\end{array}$ & $\begin{array}{l}\text { Validation } \\
\text { Group }\end{array}$ \\
\hline No & $259(85.20)$ & $115(87.12)$ \\
\hline Operative time (min) & $150.53 \pm 45.52$ & $163.27 \pm 49.33$ \\
\hline
\end{tabular}

Abbreviations: BMI, body mass index; IPP, intravesicular protrusion of the prostate; ISD, narrowest distance between the tips of the ischial spines; ISUP, International Society of Urological Pathology; ITD, widest distance between the ischial tuberosities; MAD, anteroposterior diameter of the pelvis at the mid-plane; NVB, neurovascular bundle; OAD, anteroposterior diameter of the pelvic outlet; PAD, depth of the prostatic apex; P/R: ratio of the cross-sectional areas of the prostate and the Retzius space; tPSA, total prostate-specific antigen.

Using these parameters, we defined a new pelvic size parameter: ISD/PAD, which reflects the overall size of the pelvic cavity at the level of the prostate (Figure 2).

\section{Statistical Analysis}

Statistical analysis was performed using SPSS 19.0 software (IBM, Inc., Armonk, NY, USA). Univariate and multivariate linear regression analysis were performed to determine the predictors of the duration of LRP in the model creation group. The scoring system was created using these predictors. The predictive accuracy of the scoring system was determined using receiver operating characteristic (ROC) curves. The Hosmer-Lemeshow test was used to test the goodness-of-fit of each model and calibration plots were created for visual assessment. $P<$ 0.05 was considered to represent statistical significance.

\section{Results}

The characteristics of patients in the model creation and validation groups are presented in Table 1. Univariate linear regression analysis showed that BMI, prostate volume, IPP, P/R, pelvic lymph node dissection, neurovascular bundle (NVB) preservation, and a transperitoneal approach were significant predictors of prolonged surgery (Table 2). Multivariate linear regression analysis showed that BMI, prostate volume, IPP, P/R, pelvic lymph node dissection, and NVB preservation were significant predictors of prolonged surgery (Table 3 ).

We devised a scoring system using a combination of the above six prognostic parameters, which had a total score of 6 (Table 4). Patients were classified into three groups as follows: a low-score group (0-2), an intermediate score group (3-4), and a high score group (5-6). ROC 


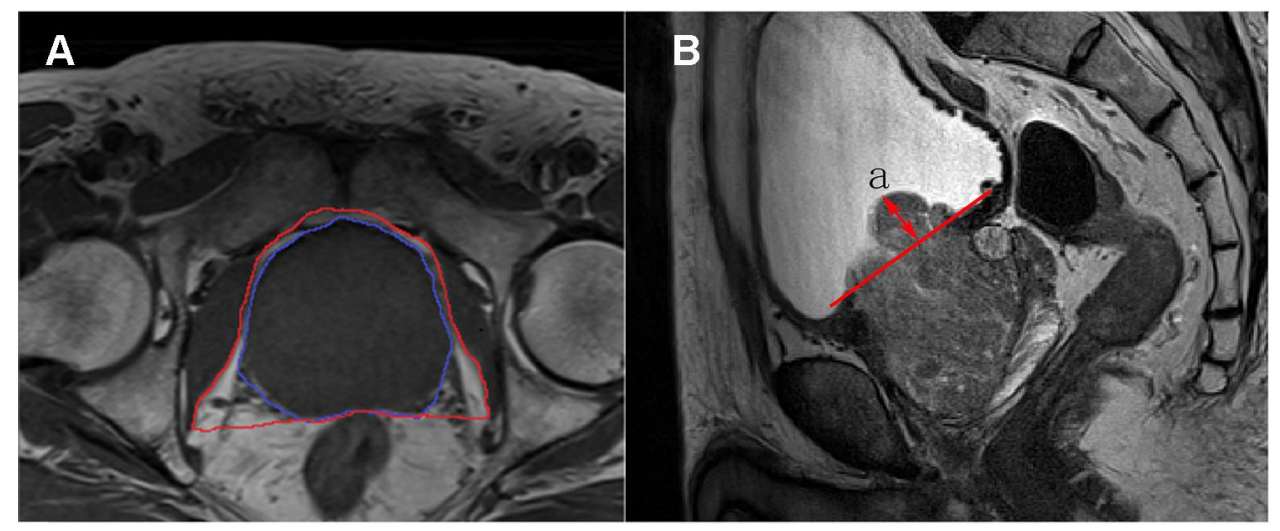

Figure I (A) The cross-sectional area of the Retzius space was defined as the area surrounded by the pubic bone, levator ani, and rectum on an MRI image, as shown by the red line. The cross-sectional area of the prostate is shown by the blue line. (B) The sagittal section of prostate on an MRI image. (a) IPP: the distance from the deepest part of prostatic protrusion into the bladder to the level of the bladder neck on the sagittal plane of the MRI image. The red double arrow refers to IPP.

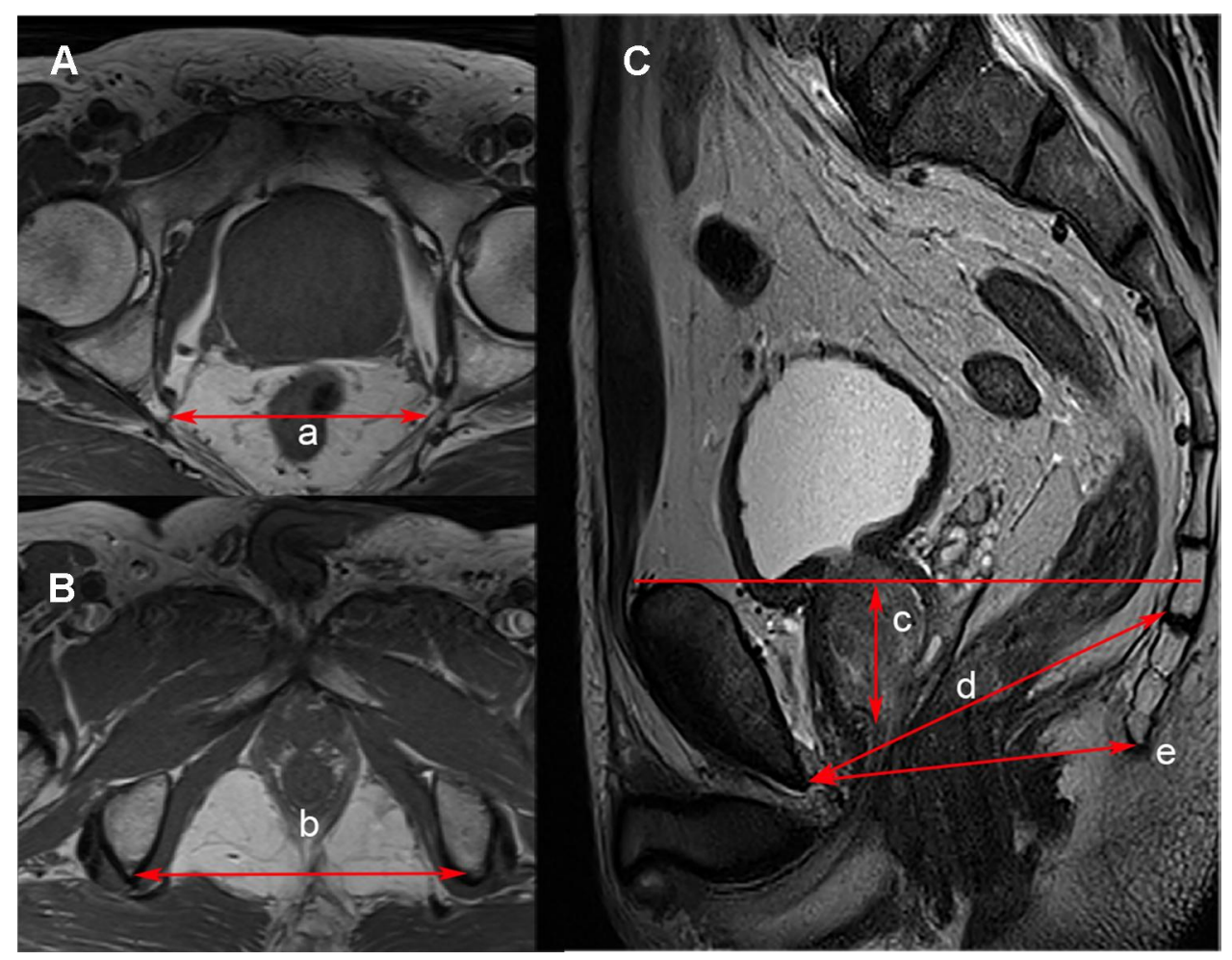

Figure 2 (A, B)The cross section and (C) sagittal section of prostate on an MRI image. (a) ISD: the narrowest distance between the tips of the ischial spines; (b) ITD: the widest distance between the ischial tuberosities at the pelvic outlet; (c) PAD: the vertical distance from the closest edge of the pubic symphysis to the distal edge of the prostatic tip on the sagittal plane of the MRI image; (d) MAD: the anteroposterior diameter of the pelvis at the mid-plane; and (e) OAD: the anteroposterior diameter of the pelvic outlet (distances indicated with red arrows).

curve analysis generated an area under the curve (AUC) for this scoring system (AUC $=0.874,95 \%$ confidence interval [CI]: $0.836-0.913$ ) that was much higher than those for BMI (AUC $=0.813,95 \%$ CI: $0.764-0.863$ ), prostate volume $(\mathrm{AUC}=0.823,95 \% \mathrm{CI}: 0.774-0.871)$, IPP (AUC $=0.792,95 \%$ CI: $0.740-0.844), \mathrm{P} / \mathrm{R} \quad(\mathrm{AUC}=0.782$, 95\% CI: $0.730-0.834)$, pelvic lymph node dissection (AUC $=0.567,95 \%$ CI: $0.50-0.631$ ), or NVB preservation
(AUC $=0.615,95 \%$ CI: $0.552-0.678)$ alone (Table 5 and Figure 3A). The Hosmer-Lemeshow goodness-of-fit test showed that the scoring system was well-calibrated $\left(\mathrm{X}^{2}=5.339, P=0.376\right)$. The observed expected plot is shown as Figure 3B.

External validation of the scoring system was performed using the independent dataset from the validation group. When the scoring system model was applied to the 
Table 2 Univariate Linear Regression Analysis of the Factors Potentially Affecting the Total Duration of Surgery

\begin{tabular}{|l|l|l|}
\hline Variables & $\mathbf{t}$ & $\mathbf{p}$ \\
\hline Age & -0.074 & 0.941 \\
BMI & 13.505 & $<0.001$ \\
Hypertension & -0.081 & 0.935 \\
Diabetes & -0.455 & 0.649 \\
tPSA & -4.65 & 0.642 \\
Prostate volume & 17.817 & $<0.001$ \\
CT Stage & 0.764 & 0.431 \\
Previous abdominal surgery & 0.423 & 0.673 \\
ISUP Groupe & -1.200 & 0.231 \\
Surgical approach & 2.361 & 0.019 \\
(transperitoneal vs extraperitoneal) & & \\
Pelvic lymph node dissection & 3.390 & 0.001 \\
NVB preservation & 4.828 & $<0.001$ \\
IPP & 14.590 & $<0.001$ \\
P/R & 9.853 & $<0.001$ \\
ISD & 0.619 & 0.536 \\
ITD & 1.699 & 0.090 \\
MAD & -0.445 & 0.657 \\
OAD & -1.726 & 0.085 \\
PAD & 0.432 & 0.666 \\
ISD/PAD & -0.455 & 0.649 \\
\hline
\end{tabular}

Abbreviations: BMI, body mass index; IPP, intravesicular protrusion of the prostate; ISD, narrowest distance between the tips of the ischial spines; ISUP, International Society of Urological Pathology; ITD, widest distance between the ischial tuberosities; MAD, anteroposterior diameter of the pelvis at the mid-plane; NVB, neurovascular bundle; OAD, anteroposterior diameter of the pelvic outlet: $P A D$, depth of the prostatic apex; P/R: ratio of the cross-sectional areas of the prostate and the Retzius space; tPSA, total prostate-specific antigen.

validation cohort, we observed a high level of predictive accuracy (AUC=0.835, 95\% CI: 0.764-0.906, Figure 4A). The Hosmer-Lemeshow goodness-of-fit test showed that the scoring system was also well calibrated for this group $\left(\mathrm{X}^{2}=4.401, P=0.493\right)$, and the observed expected plot is shown as Figure 4B.

\section{Discussion}

In China, the prevalence of prostate cancer has shown a rapid upward trend in recent years. Indeed, in some developed cities, it even exceeds that of bladder cancer, making it the most common malignant genitourinary tumor in men. ${ }^{16}$ Schuessler et al ${ }^{17}$ first officially reported the use of LRP in 1997, but as the technology has matured and the popularity of laparoscopic equipment has risen, it has become the standard procedure for the treatment of localized prostate cancer. Although RALP has been widely used in Europe and the United States, LRP remains the standard treatment for localized prostate cancer in China.

Huang et $\mathrm{al}^{18}$ found that prolonged radical prostatectomy results in prolonged hospitalization, which causes a significant increase in hospital costs, and more importantly, increases the risk of postoperative complications. Although previous studies have identified predictors of prolonged radical prostatectomy, none attempted to accurately predict the duration of the procedure. ${ }^{15,19,20}$ There are many factors that influence LRP, which can be divided into the following three categories: (1) Objective clinical characteristics, such as age, the underlying disease, the pathological characteristics, and local anatomy; (2) Surgical method, such as the surgical approach, the lymph node dissection procedure, and whether NVBs are preserved; and (3) The proficiency of the surgeon with respect to the procedure. In order to achieve a more precise estimate of the duration of the procedure, we retrospectively analyzed the perioperative data associated with the participants to identify predictors of the duration of LRP.

Our data show that BMI positively correlates with the duration of LRP, which is consistent with previous reports.

Table 3 Multivariate Linear Regression Analysis of Factors Potentially Affecting the Total Duration of Surgery

\begin{tabular}{|l|l|l|l|l|l|}
\hline \multirow{2}{*}{ Variables } & \multicolumn{2}{|l|}{ Unstandardized Coefficients } & Standardized Coefficients & t & P \\
\cline { 2 - 5 } & $\boldsymbol{\beta}$ & Std. Error & Beta & \\
\hline (Constant) & -35.084 & 16.553 & & -2.120 & 0.035 \\
BMI & 2.727 & 0.712 & 0.174 & 3.830 & $<0.001$ \\
Prostate volume & 1.012 & 0.112 & 0.408 & 9.000 & $<0.001$ \\
Surgical approach (transperitoneal vs extraperitoneal) & 4.291 & 3.791 & 0.041 & 1.132 & 0.259 \\
Pelvic lymph node dissection & 7.961 & 3.650 & 0.081 & 2.181 & 0.030 \\
NVB preservation & 9.887 & 3.586 & 0.099 & 0.006 \\
IPP & 21.220 & 5.013 & 0.199 & 2.757 & 0.233 \\
P/R & 63.728 & 18.827 & 0.133 & $<0.001$ \\
\hline
\end{tabular}

Abbreviations: BMI, body mass index; IPP, intravesicular protrusion of the prostate; NVB, neurovascular bundle; P/R, ratio of the cross-sectional areas of the prostate and the Retzius space. 
Table 4 Scoring System for the Prediction of Prolonged LRP

\begin{tabular}{|c|c|c|}
\hline \multicolumn{2}{|l|}{ Parameters } & \multirow{2}{*}{$\begin{array}{l}\text { Score } \\
1 \\
0\end{array}$} \\
\hline BMI $\left(\mathrm{Kg} / \mathrm{m}^{2}\right)$ & $\begin{array}{l}\geq 24.12 \\
<24.12\end{array}$ & \\
\hline Prostate volume $(\mathrm{mL})$ & $\begin{array}{l}\geq 44.32 \\
<44.32\end{array}$ & $\begin{array}{l}1 \\
0\end{array}$ \\
\hline Pelvic lymph node dissection & $\begin{array}{l}\text { Yes } \\
\text { No }\end{array}$ & $\begin{array}{l}1 \\
0\end{array}$ \\
\hline NVB preservation & $\begin{array}{l}\text { Yes } \\
\text { No }\end{array}$ & $\begin{array}{l}1 \\
0\end{array}$ \\
\hline IPP (mm) & $\begin{array}{l}\geq 8.52 \\
<8.52\end{array}$ & $\begin{array}{l}1 \\
0\end{array}$ \\
\hline$P / R$ & $\begin{array}{l}\geq 0.68 \\
<0.68\end{array}$ & $\begin{array}{l}1 \\
0\end{array}$ \\
\hline
\end{tabular}

Abbreviations: BMI, body mass index; NVB, neurovascular bundle; P/R, ratio of the cross-sectional areas of the prostate and the Retzius space.

Table 5 The AUC of the ROC Curves for Factors Predictive of Prolonged LRP

\begin{tabular}{|l|l|l|l|}
\hline Parameters & AUC & 95\% CI & P \\
\hline Scoring system & 0.874 & $0.836-0.913$ & $<0.00 I$ \\
BMI & 0.813 & $0.764-0.863$ & $<0.00 I$ \\
Prostate volume & 0.823 & $0.774-0.87 I$ & $<0.00 I$ \\
Pelvic lymph node dissection & 0.567 & $0.502-0.631$ & 0.045 \\
NVB preservation & 0.615 & $0.552-0.678$ & $0.00 I$ \\
IPP & 0.792 & $0.740-0.844$ & $<0.00 I$ \\
P/R & 0.782 & $0.730-0.834$ & $<0.00 I$ \\
\hline
\end{tabular}

Abbreviations: BMI, body mass index; IPP, intravesicular protrusion of the prostate; NVB, neurovascular bundle; P/R, ratio of the cross-sectional areas of the prostate and the Retzius space.

Kaneko et $\mathrm{al}^{15}$ measured the thickness of the perirectal fat tissue in 50 patients by transrectal ultrasonography, and found that patients with a high BMI have abundant perirectal fat, which prolongs the dissection of the seminal vesicle and the vas deferens, as well as the separation of the prostate from the rectum. Patients with high BMI usually have a high body fat content. Adipose tissue can promote the conversion of androgens into estrogen, which can cause the proliferation of interstitial cells, and eventually an increase in prostate volume. ${ }^{21}$ Several studies have shown that compared with normal-weight prostate cancer patients, obese patients have a higher incidence of extracapsular tumor extension: overweight patients were about twice as likely, and obese patients were up to four times as likely to show this. ${ }^{22}$ This is important, because extracapsular tumor extension may cause adhesions between the prostate and surrounding tissues, thereby making dissection more difficult and prolonging the duration of the procedure.

In addition to high BMI, we also found that larger prostate volume is an independent predictor of prolonged surgery. Pettus et al found that an enlarged prostate increases the risk of intraoperative bleeding, which prolongs surgery. ${ }^{23}$ In addition, Kaneko et al reported that during blunt dissection between the prostate and levator ani, an enlarged prostate reduces the available workspace, which makes dissection of the seminal vesicle and the vas deferens, and the separation of the prostate and rectum more difficult, thereby prolonging the procedure. ${ }^{15}$

In order to evaluate the size of the space around the prostate as objectively as possible, we defined the ratio of the cross-sectional area of the prostate to that of the Retzius space (P/R: prostate/Retzius space), and found that the duration of LRP increased with an increase in this ratio. As shown in Figure 1A, the larger the ratio, the narrower the space around the prostate, and the more difficult the procedure. Because the Retzius space contains a venous plexus, narrowing increases the risk of hemorrhage, which further prolongs surgery.

Singh et $\mathrm{al}^{24}$ suggested that pelvic size may be an important factor affecting LRP, and in the current era of laparoscopic and robot-assisted prostatectomy, pelvic size may be even more important, because the space in which instruments must be manipulated during pelvic surgery is more restricted anyway. Some previous studies have shown that it is more challenging to perform lateral and posterior dissection of the prostate in a narrow pelvis using a laparoscopic technique. Therefore, we measured various pelvic parameters (ISD, ITD, MAD, OAD, and PAD) in the present study. PAD represents a useful assessment of the pelvic depth in the surgical field, especially during apical manipulations, such as dorsal vein ligation, apical dissection, and urethral anastomosis. In addition, we used the ISD/PAD ratio to indirectly assess the pelvic space at the level of the prostate. Patients with a wide, shallow pelvis have a higher ISD/PAD, whereas those with a narrow, deep pelvis have a relatively low ISD/PAD. However, none of these parameters were statistically significant predictors of the duration of LRP. It is possible that a difference in pelvic size is just one of the sources of anatomical variation in the male pelvis, and therefore has a limited impact on LRP, and indeed we found that the variations in pelvic size were not as significant as those in BMI or prostate volume. For example, the difference 


\section{ROC curve}

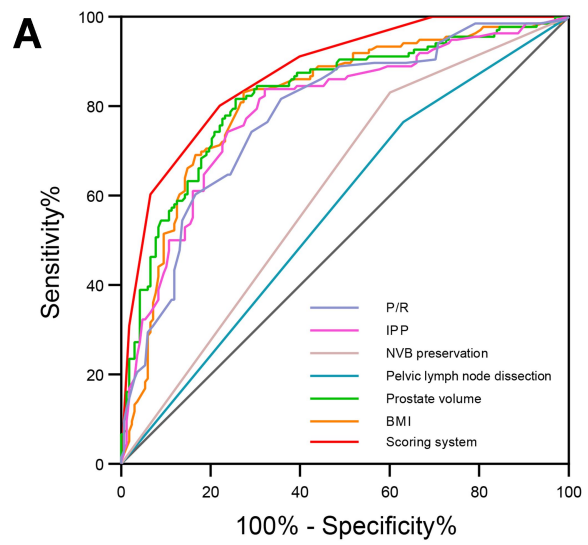

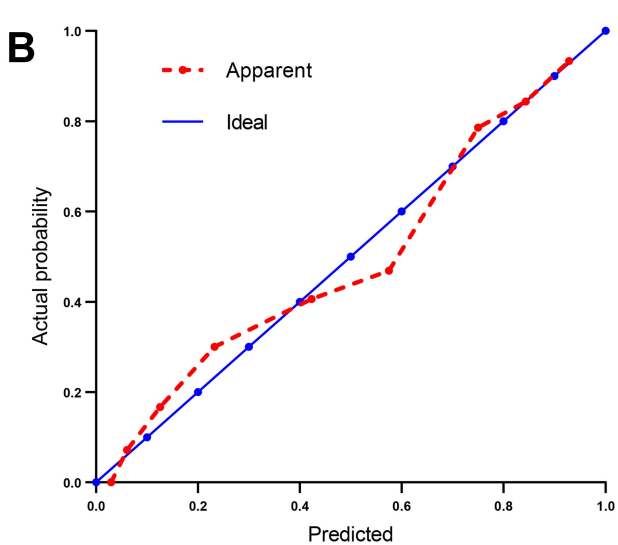

Figure 3 (A) ROC curve analysis of the novel scoring system and other factors that were predictors of the duration of surgery; and (B) Hosmer-Lemeshow goodness-of-fit test results, showing good calibration for the scoring system $\left(X^{2}=5.339, P=0.376\right)$.

\section{ROC curve: External Validation}

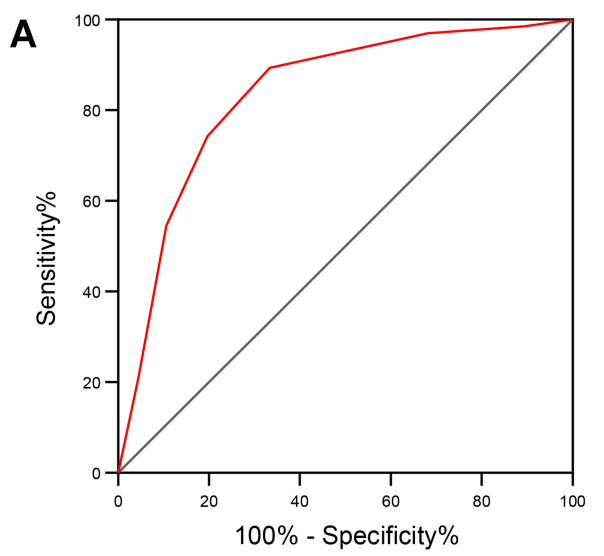

B

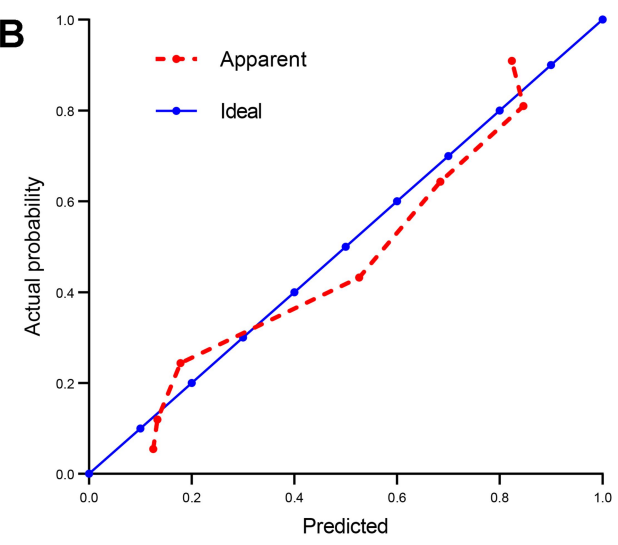

Figure 4 External validation of the scoring system. (A) The novel scoring system achieved good predictive accuracy in the validation cohort. (B) The Hosmer-Lemeshow goodness-of-fit test also showed good calibration for the scoring system in this cohort $\left(X^{2}=4.401, P=0.493\right)$.

between the maximum and minimum values of ISD was only about $3 \mathrm{~cm}$, which might explain why it has no significant effect on the duration of LRP. Therefore, pelvic size is not an important factor determining the duration of surgery for experienced surgeons, compared with other parameters.

In previous studies, IPP has long been used as an indicator of bladder outlet obstruction in patients with benign prostatic hyperplasia. ${ }^{25,26}$ However, the relationship between IPP and prostate cancer has rarely been discussed. In the present study, we found that IPP positively correlates with the duration of LRP, and is an important predictor of prolonged surgery. In most patients with a large IPP, hyperplasia of the lateral and median lobes leads to prostatic protrusion into the bladder, a change in the ureteral orifice, and potentially bladder outlet obstruction, which increase the risk of bleeding during the separation of the prostate from the bladder neck and the difficulty of reconstructing the bladder neck. Therefore, it is logical that substantial IPP prolongs LRP.

A retrospective study conducted by Hoznek et $\mathrm{al}^{27}$ found that in comparison to the transperitoneal laparoscopic approach, the extraperitoneal laparoscopic technique was much faster (169.6 min vs $224.2 \mathrm{~min}, P<0.001)$. Cathelineau et al and Ruiz et al also showed that the extraperitoneal approach reduced the duration of the procedure, because it allows direct access to the Retzius space. During transperitoneal procedures, more time is needed to create a rectovesical space, especially in 
obese patients and patients that have undergone previous abdominal surgery. ${ }^{28,29}$ However, several studies have shown that there is no significant difference in the duration of surgery between the two approaches..$^{28,30,31}$ In the present study, univariate linear regression analysis showed that a transperitoneal approach was a significant predictor of prolonged surgery, but multivariate linear regression analysis excluded this as independent predictive factor.

Recent studies have shown that NVB preservation during LRP is conducive to the preservation and recovery of sexual function and an improvement in urination after surgery. ${ }^{32}$ If NVB preservation is required, attention should be paid to the base of the prostate: the thin fascia covering the neurovascular bundle should be carefully incised and the NVB should be released from the base of the prostate to the pelvic floor muscle on the posterolateral side of the urethra using blunt and sharp dissection. In the present study, LRP lasted about 30 min longer if the NVB was preserved than if it was not (159.5 \pm 44.9 vs $129.2 \pm 39.2 \mathrm{~min}$ ).

Simon et al identified several predictors of the durations of radical retropubic prostatectomy or RALP and also created corresponding nomograms for the prediction of the duration of surgery. They found that BMI, race, prostate mass, lymph node dissection, and hospital surgical volume were all predictors of the duration of radical retropubic prostatectomy, whereas biopsy Gleason score, lymph node dissection, and hospital surgical volume were predictors of the duration of robot-assisted laparoscopic prostatectomy. ${ }^{4}$ Furthermore, Kaneko et al reported that BMI and prostate mass predicted longer LRP. However, few factors were assessed in this study and no more precise estimate of the duration of LRP was made. ${ }^{15}$

To date, there have been no reports of scoring systems that could predict the duration of LRP in China or elsewhere. In the present study, multivariate linear regression analysis showed that BMI, prostate volume, IPP, P/R, pelvic lymph node dissection, and NVB preservation are independent predictors of prolonged surgery. Using a combination of these predictors, we have created a scoring system to predict the duration of LRP, which appears to be of great value. As Violette and colleagues put it, "knowledge of the predictors may assist in surgical planning and improve resource utilization". ${ }^{20}$ Estimation of the duration of a procedure can help the surgeons and nurses improve preoperative preparation and perioperative efficiency.

There were some limitations to the study. First, it was a single-center, retrospective study and the sample size was relatively small, with only 436 patients included. Moreover, the scoring system was not externally validated and its predictive value in other populations remains to be established. In addition, the factors influencing the duration of LRP are relatively complex; therefore, the inclusion of more indicators may further improve the predictive model.

\section{Conclusions}

In conclusion, $\mathrm{BMI}$, prostate volume, IPP, $\mathrm{P} / \mathrm{R}$, pelvic lymph node dissection, and NVB preservation are significant predictors of prolonged LRP. We have created a novel scoring system that can accurately predict the duration of surgery, assess its difficulty, and improve perioperative efficiency.

\section{Abbreviations}

BMI, body mass index; IPP, intravesicular protrusion of the prostate; ISD, narrowest distance between the tips of the ischial spines; ISUP, International Society of Urological Pathology; ITD, widest distance between the ischial tuberosities; LRP, arthroscopic radical prostatectomy; MAD, anteroposterior diameter of the pelvis at the mid-plane; NVB, neurovascular bundle; OAD, anteroposterior diameter of the pelvic outlet; PAD, depth of the prostatic apex; $\mathrm{P} / \mathrm{R}$, ratio of the cross-sectional areas of the prostate and the Retzius space; RALP, robot-assisted laparoscopic prostatectomy; RP, radical prostatectomy; tPSA, total prostate-specific antigen.

\section{Ethics Approval and Informed Consent}

This study was approved by the Ethics Committee of the First Affiliated Hospital of Fujian Medical University (No. 2,015,084). All patients have provided written informed consent.

\section{Funding}

This study was supported by Startup Fund for scientific research, Fujian Medical University (Grant number: 2017XQ1083), the Young and Middle-aged Talents Training Project of Fujian Provincial Health Commission (Grant number: 2019-ZQN-54) and Foundation of Fujian Provincial Department of Finance (Grant number: 2018B011).

\section{Disclosure}

The authors report no conflicts of interest for this work. 


\section{References}

1. Allott EH, Abern MR, Gerber L, et al. Metformin does not affect risk of biochemical recurrence following radical prostatectomy: results from the SEARCH database. Prostate Cancer Prostatic Dis. 2013;16(4):391-397. doi:10.1038/pcan.2013.48

2. Xu N, Wu YP, Yin HB, et al. SHCBP1 promotes tumor cell proliferation, migration, and invasion, and is associated with poor prostate cancer prognosis. J Cancer Res Clin Oncol. 2020;146(8):1953-1969. doi:10.1007/s00432-020-03247-1

3. Xu N, Wu YP, Li XD, et al. Risk of upgrading from prostate biopsy to radical prostatectomy pathology: is magnetic resonance imaging-guided biopsy more accurate? J Cancer. 2018;9 (19):3634-3639. doi:10.7150/jca.26791

4. Simon RM, Howard LE, Moreira DM, et al. Predictors of operative time during radical retropubic prostatectomy and robot-assisted laparoscopic prostatectomy. Int $J$ Urol. 2017;24(8):618-623. doi:10.1111/iju. 13393

5. Eijkemans MJ, van Houdenhoven M, Nguyen T, Boersma E, Steyerberg EW, Kazemier G. Predicting the unpredictable: a new prediction model for operating room times using individual characteristics and the surgeon's estimate. Anesthesiology. 2010;112 (1):41-49. doi:10.1097/ALN.0b013e3181c294c2

6. Dexter F. A strategy to decide whether to move the last case of the day in an operating room to another empty operating room to decrease overtime labor costs. Anesth Analg. 2000;91(4):925-928. doi:10.1097/00000539-200010000-00029

7. Hammermeister K. The national surgical quality improvement program: learning from the past and moving to the future. Am J Surg. 2009;198(5 Suppl):S6973. doi:10.1016/j.amjsurg.2009.08.007

8. Mikhail AA, Stockton BR, Orvieto MA, et al. Robotic-assisted laparoscopic prostatectomy in overweight and obese patients. Urology. 2006;67(4):774-779. doi:10.1016/j.urology.2005.10.049

9. Herman MP, Raman JD, Dong S, Samadi D, Scherr DS. Increasing body mass index negatively impacts outcomes following robotic radical prostatectomy. JSLS. 2007;11(4):438-442.

10. Yasui T, Tozawa K, Kurokawa S, et al. Impact of prostate weight on perioperative outcomes of robot-assisted laparoscopic prostatectomy with a posterior approach to the seminal vesicle. BMC Urol. 2014;14:6. doi:10.1186/1471-2490-14-6

11. Martinez CH, Chalasani V, Lim D, et al. Effect of prostate gland size on the learning curve for robot-assisted laparoscopic radical prostatectomy: does size matter initially? J Endourol. 2010;24(2):261-266. doi:10.1089/end.2009.0325

12. Yong DZ, Tsivian M, Zilberman DE, Ferrandino MN, Mouraviev V, Albala DM. Predictors of prolonged operative time during robot-assisted laparoscopic radical prostatectomy. BJU Int. 2011;107(2):280-282. doi:10.1111/j.1464-410X.2010.09511.x

13. Zilberman DE, Tsivian M, Yong D, Albala DM. Surgical steps that elongate operative time in robot-assisted radical prostatectomy among the obese population. $J$ Endourol. 2011;25(5):793-796. doi:10.1089/end.2010.0428

14. Ploussard G, Briganti A, de la Taille A, et al. Pelvic lymph node dissection during robot-assisted radical prostatectomy: efficacy, limitations, and complications-a systematic review of the literature. Eur Urol. 2014;65(1):7-16. doi:10.1016/j.eururo.2013.03.057

15. Kaneko G, Miyajima A, Yazawa S, et al. What is the predictor of prolonged operative time during laparoscopic radical prostatectomy? Int J Urol. 2013;20(3):330-336. doi:10.1111/j.1442-2042.2012.03185.x

16. Liu X, Yu C, Bi Y, Zhang ZJ. Trends and age-period-cohort effect on incidence and mortality of prostate cancer from 1990 to 2017 in China. Public Health. 2019;172:70-80. doi:10.1016/j.puhe.2019. 04.016
17. Schuessler WW, Schulam PG, Clayman RV, Kavoussi LR. Laparoscopic radical prostatectomy: initial short-term experience. Urology. 1997;50(6):854-857. doi:10.1016/S0090-4295(97)00543-8

18. Huang KH, Kaplan AL, Carter SC, Lipsitz SR, Hu JC. The impact of radical prostatectomy operative time on outcomes and costs. Urology. 2014;83(6):1265-1271. doi:10.1016/j.urology.2014.01.047

19. El-Feel A, Davis JW, Deger S, et al. Laparoscopic radical prostatectomy-an analysis of factors affecting operating time. Urology. 2003;62(2):314-318. doi:10.1016/S0090-4295(03)00250-4

20. Violette PD, Mikhail D, Pond GR, Pautler SE. Independent predictors of prolonged operative time during robotic-assisted radical prostatectomy. J Robot Surg. 2015;9(2):117-123. doi:10.1007/ s11701-015-0497-0

21. Loeb S, Yu X, Nadler RB, et al. Does body mass index affect preoperative prostate specific antigen velocity or pathological outcomes after radical prostatectomy? J Urol. 2007;177(1):102-106; discussion 106.

22. Davies BJ, Smaldone MC, Sadetsky N, Dall'era M, Carroll PR. The impact of obesity on overall and cancer specific survival in men with prostate cancer. J Urol. 2009;182(1):112-117; discussion 117. doi:10.1016/j.juro.2009.02.118

23. Pettus JA, Masterson T, Sokol A, et al. Prostate size is associated with surgical difficulty but not functional outcome at 1 year after radical prostatectomy. J Urol. 2009;182(3):949-955. doi:10.1016/j. juro.2009.05.029

24. Singh A, Fagin R, Shah G, Shekarriz B. Impact of prostate size and body mass index on perioperative morbidity after laparoscopic radical prostatectomy. J Urol. 2005;173(2):552-554. doi:10.1097/01. ju.0000150101.95236.35

25. Kadihasanoglu M, Aydin M, Taskiran M, Kendirci M. The effect of intravesical prostatic protrusion in patients with benign prostatic hyperplasia: controlled, clinical study. Urol Int. 2019;103 (2):180-186. doi:10.1159/000499437

26. Kalkanli A, Tandogdu Z, Aydin M, et al. Intravesical prostatic protrusion: a potential marker of alpha-blocker treatment success in patients with benign prostatic enlargement. Urology. 2016;88:161-165. doi:10.1016/j.urology.2015.11.029

27. Hoznek A, Antiphon P, Borkowski T, et al. Assessment of surgical technique and perioperative morbidity associated with extraperitoneal versus transperitoneal laparoscopic radical prostatectomy. Urology. 2003;61(3):617-622. doi:10.1016/S0090-4295(02)02415-9

28. Cathelineau X, Cahill D, Widmer H, Rozet F, Baumert H, Vallancien G. Transperitoneal or extraperitoneal approach for laparoscopic radical prostatectomy: a false debate over a real challenge. $J \quad$ Urol. 2004;171(2 Pt 1):714-716. doi:10.1097/01. ju.0000103885.71434.02

29. Ruiz L, Salomon L, Hoznek A, et al. Comparison of early oncologic results of laparoscopic radical prostatectomy by extraperitoneal versus transperitoneal approach. Eur Urol. 2004;46(1):50-54; discussion 54-56. doi:10.1016/j.eururo.2004.04.013

30. Erdogru T, Teber D, Frede T, et al. Comparison of transperitoneal and extraperitoneal laparoscopic radical prostatectomy using match-pair analysis. Eur Urol. 2004;46(3):312-319; discussion 320. doi:10.1016/j.eururo.2004.05.004

31. Brown JA, Rodin D, Lee B, Dahl DM. Transperitoneal versus extraperitoneal approach to laparoscopic radical prostatectomy: an assessment of 156 cases. Urology. 2005;65(2):320-324. doi:10.1016/j. urology.2004.09.018

32. de Carvalho PA, Barbosa J, Guglielmetti GB, et al. Retrograde release of the neurovascular bundle with preservation of dorsal venous complex during robot-assisted radical prostatectomy: optimizing functional outcomes. Eur Urol. 2018. 


\section{Publish your work in this journal}

Cancer Management and Research is an international, peer-reviewed open access journal focusing on cancer research and the optimal use of preventative and integrated treatment interventions to achieve improved outcomes, enhanced survival and quality of life for the cancer patient.

The manuscript management system is completely online and includes a very quick and fair peer-review system, which is all easy to use. Visit http://www.dovepress.com/testimonials.php to read real quotes from published authors. 